\title{
Germanica
}

\section{L'horizon utopique d'une totalité des langues et cultures Plurilinguisme et écriture plurilingue chez Ilma Rakusa}

The utopian horizon of a totality of languages and cultures: multilingualism and multilingual writing in the works of Ilma Rakusa

Der utopische Horizont einer Totalität der Sprachen und Kulturen :

Mehrsprachigkeit und mehrsprachiges Schreiben bei Ilma Rakusa

\section{Dirk Weissmann}

\section{(2) OpenEdition}

\section{Journals}

Édition électronique

URL : http://journals.openedition.org/germanica/2014

DOI : 10.4000/germanica.2014

ISSN : 2107-0784

\section{Éditeur}

Université de Lille

\section{Édition imprimée}

Date de publication : 31 décembre 2012

Pagination : 149-163

ISBN : 9782913857308

ISSN : 0984-2632

\section{Référence électronique}

Dirk Weissmann, «L'horizon utopique d'une totalité des langues et cultures Plurilinguisme et écriture plurilingue chez IIma Rakusa », Germanica [En ligne], 51 | 2012, mis en ligne le 05 février 2013, consulté le 06 octobre 2020. URL : http://journals.openedition.org/germanica/2014 ; DOI : https://doi.org/

10.4000/germanica.2014

Ce document a été généré automatiquement le 6 octobre 2020.

(C) Tous droits réservés 


\section{L'horizon utopique d'une totalité des langues et cultures Plurilinguisme et écriture plurilingue chez Ilma Rakusa}

The utopian horizon of a totality of languages and cultures: multilingualism and multilingual writing in the works of Ilma Rakusa

Der utopische Horizont einer Totalität der Sprachen und Kulturen :

Mehrsprachigkeit und mehrsprachiges Schreiben bei Ilma Rakusa

Dirk Weissmann

Et donc on devient poète [...] pour être non pas français, russe, etc., mais pour être tout. Ou encore : on est poète parce qu'on n'est pas français. La nationalité est forclusion et inclusion. Orphée fait éclater la nationalité ou l'élargit à tel point que tous (présents et passés) y sont inclus ${ }^{1}$.

\section{«Transit. Transfinit » ${ }^{2}$}

1 On pourrait dire qu'une dialectique fondamentale sous-tend l'œuvre d'Ilma Rakusa, écrivaine, traductrice et essayiste, celle entre la frontière et l'ouverture, entre le propre et l'étranger, entre l'unité et la multiplicité. La tension entre ces pôles, tendant vers une totalité que l'écrivain sait pourtant inatteignable, se condense dans le mot de «transfini » (transfinit) employé par elle-même. À travers ce terme emprunté aux mathématiques s'exprime la synthèse (paradoxale) entre un lieu de passage et une entité achevée. Dressant son autoportrait pour inaugurer le cycle de conférences données dans le cadre de la Dresdner Poetikdozentur (« leçons de littérature »), en 2005, elle se définit comme une « passeuse de frontières » : 
La frontière sensibilise à la diversité et à la tension entre le dedans et le dehors, entre le familier et l'étranger, entre le proche et le lointain. Je suis reconnaissante de cette sensibilisation. Car elle m'a sans aucun doute conduite à devenir une passeuse de frontières, à travers l'écriture, et une traductrice. La traversée des frontières entre les langues et les Etats est devenue mon école de la vie; le frottement entre le propre et l'étranger mon stimulus artistique. J'ai vécu la frontière dans son ambivalence (productrice) : comme barrière et pont à la fois ${ }^{3}$.

2 Il en résulte une mobilité permanente de l'écriture, de l'esprit et du corps, toujours enclins à se transporter ailleurs. "Je est multiple $»^{4}$, dit-elle, en paraphrasant Rimbaud. Dans ses textes, les longs voyages en train, la topographie des gares, villes et paysages lointains et inconnus deviennent les emblèmes de cette existence transitoire où l'espace se rétracte, le présent et le passé se télescopent : "Je suis ici et en même temps ailleurs $»^{5}$.

3 Du point de vue de sa langue - ou plutôt de ses langues, car Ilma Rakusa en possède ou pratique plus d'une demi-douzaine -, elle se décrit comme viscéralement polyglotte : « Il y des jours où je soliloque en cinq langues et où je ressens que mon écriture aimerait également utiliser ces cinq langues. Prends le meilleur de tout [...] et crée ton propre idiome à travers l'alternance des langues ${ }^{6}$. $"$ Or, malgré ce désir d'une écriture quasi babélienne ${ }^{7}$, Rakusa s'accroche à son identité d'écrivaine de langue allemande, seul ancrage stable qu'elle arrive à trouver. Ainsi, pour parler de son identité, elle ne propose qu'une définition "poétique ", posant la langue (allemande) et la littérature comme seule patrie ${ }^{8}$. Ainsi, refusant toute une série d'étiquettes (nationales) qu'on a essayé de lui attribuer, elle affirme : «Je ne suis rien d'autre qu'une écrivaine de langue allemande qui vit a Zurich ${ }^{9}$."

4 Malgré cet attachement à l'allemand, le plurilinguisme - comme réalité sociale, pratique littéraire et figure de pensée à la fois - est indissociable de son œuvre. Car, cette langue allemande n'est pas sa langue dite maternelle, même pas une langue "seconde ", mais une langue apprise relativement tard, après bien d'autres. "Je n'ai qu'une langue, or elle est multiple », pourrait-on lui faire dire, en variant le propos de Jacques Derrida dans Le monolinguisme de l'autre $e^{10}$. Ainsi, ses œuvres, écrites en allemand, sont traversées par le hongrois, le slovène, le serbo-croate, le russe, l'italien, le français, l'anglais..., tous plus ou moins liés à sa biographie et à sa formation.

\section{Une socialisation multiculturelle et plurilingue}

Née en 1946 en (Tchéco-)Slovaquie, d'un père slovène et d'une mère hongroise, Ilma Rakusa évolue d'emblée dans un univers multiculturel et plurilingue. Parmi les étapes de son enfance, on peut citer Rimavská Sobota, sa ville natale slovaque, puis Budapest et Ljubljana. Mais c'est la ville de Trieste, où elle a vécu au sortir de sa prime enfance, qui l'a sans doute le plus marquée. Incarnation du mythe habsbourgeois ${ }^{11}$, Trieste symbolise cette association paradoxale entre unité et altérité, ce "chez-soi dans l'entre-deux $»^{12}$ qui caractérise toute la vie et l'écriture d'Ilma Rakusa. Ville maritime, elle devient le lieu emblématique de l'autobiographie de l'écrivaine, intitulée Mehr $\mathrm{Meer}^{13}$.

6 Cependant, la migration familiale allait l'amener "dans le nord ${ }^{14}$, en Suisse, étape finale de son périple. Lorsque, durant sa cinquième année, sa famille s'installe à Zurich, la petite Ilma a donc déjà vécu dans une multitude de langues, transmises par sa famille 
et par son environnement. Ce qui a contribué au développement d'une conscience aiguë du relativisme des langues et cultures: "Sur quelles fondations linguistiques avancé-je ? Qu'est-ce qui distingue telle langue de telle autre, etc. ? se demande la jeune fille. Depuis toute petite, j'ai appris à comparer, sonder, jauger. J'ai perdu la croyance à la possibilité de l'un, de l'absolu, d'un fondement stable ${ }^{15}$."

7 N'arrivant qu'en quatrième lieu ${ }^{16}$, l'allemand deviendra sa langue principale ${ }^{17}$, même si la diglossie suisse ${ }^{18}$ fait que cette langue se dédouble d'emblée en Hochdeutsch et dialecte. "L'apprentissage de l'allemand ne s'est pas fait tout seul, il est dû à de longs efforts qui, cependant, m'ont ouvert beaucoup de portes ", témoigne Rakusa ${ }^{19}$. Bientôt l'écolière excellera dans cette nouvelle langue, langue d'expression de la future écrivaine, membre, depuis 1996, de la Deutsche Akademie für Sprache und Dichtung.

«Mon oreille était sensibilisée aux différentes langues : elles étaient un indicateur de richesse et de différence ", écrit-elle en parlant de son enfance ${ }^{20}$. Cette sensibilité, elle a su la cultiver et la développer à travers son parcours scolaire et professionnel. Sans cette sensibilisation précoce inscrite dans sa biographie, elle ne serait sans doute devenue ni écrivaine ni traductrice, comme elle l'affirme elle-même ${ }^{21}$. Se destinant d'abord à une carrière de musicienne professionnelle, elle commence, en 1965, des études de lettres à l'université de Zurich, avec une spécialisation dans la langue et la littérature russe et française. Ces études la mèneront à Paris (1965-1966) et à Leningrad (St. Petersburg, en 1969), où elle passera à chaque fois une année universitaire. Sans oublier les nombreux autres voyages qui jalonnent sa vie...

9 Après une thèse de doctorat achevée en 1971 et consacrée au « motif de la solitude dans la littérature russe », elle occupera pendant six ans un poste d'enseignant-chercheur au Département d'études slaves de l'université de Zurich. Si elle a gardé une charge d'enseignement dans ce département, c'est vers l'écriture et la traduction qu'elle va s'orienter à partir du milieu des années 1970 . Son premier livre, un recueil de poèmes, paraît en $1977^{22}$. Durant les années 1980, elle signe plusieurs traductions d'auteurs majeurs tels que Marguerite Duras, Marina Tsvetaïeva, Anton Tchekhov, Danilo Kiš ou Imre Kertész. Elle est également auteur d'essais critiques sur la littérature et éditrice de nombreuses anthologies. En additionnant toutes ses activités, on décèle la présence de pas moins d'une demi-douzaine de langues dans la vie et le travail d'Ilma Rakusa.

\section{« Je suis partie, pour arriver, et je suis arrivée, pour repartir $^{23}$. »}

10 Une enfance rythmée par de fréquents déménagements, une histoire familiale marquée par une pluralité linguistique et culturelle, ainsi qu'une curiosité intellectuelle la portant vers toujours plus de langues et de cultures pourraient faire d'Ilma Rakusa le parangon de l'écrivain nomade ${ }^{24}$. En effet, elle affirme elle-même n'avoir que des "racines aériennes », le sentiment d'appartenance nationale lui faisant complètement défaut ${ }^{25}$. Or, malgré le riche bagage culturel et linguistique que lui ont apporté ses années d'errance durant l'enfance, l'auteur jette également un regard critique sur cette période.

11 Le sentiment de n'avoir pas de chez-soi, de n'être nulle part à sa place, d'être ballotée, a $\mathrm{pu}$ avoir des effets traumatisants. Ainsi, parle-t-elle de son "traumatisme de la valise » : «En grandissant, je me demande qui je suis. / Les valises, même recouvertes 
de stickers aux couleurs les plus gaies, ne donnent pas de réponse à cette question. / Les valises font $\mathrm{mal}^{26}$. » La présence d'une citation implicite fait penser à la figure du Wanderer, ce personnage errant cherchant sa destinée cher aux lieder de Schubert : « Il faut continuer, toujours plus loin, je connaissais la mélodie. Sauf que j'ignorais le pourquoi de la chose. Personne ne m'a jamais demandé mon avis ${ }^{27}$. "

Ce manque de repères et ce sentiment d'insécurité datant de l'enfance explique sans doute la décision d'élire domicile dans la langue allemande afin de disposer d'un ancrage stable au milieu de tant de changements : «Après les trois langues que j'avais déjà apprises, cette quatrième était mon point de fuite et mon refuge. C'est ici que je voulais m'installer, c'est ici que j'ai construit ma maison. Elle devait être solide ${ }^{28}$. » Plus tard, alors que l'adolescente commence à écrire ses premiers poèmes, elle prend conscience que des limites et un certain ordre lui sont nécessaires au développement de sa créativité :

L'enjeu était-il la stabilité ? S'agissait-il d'un remède au nomadisme de mon enfance, avec ses changements de lieu, ses déménagements, ses incertitudes? Me fixais-je des limites à moi-même ? Pour explorer d'autres espaces, intérieurs ? ${ }^{29}$

Elle comprend que même le provincialisme de Zurich, faisant contrepoids à son désir des lointains, lui est dans un certain sens profitable :

À cette époque m'est venue l'idée que Zurich me demandait une ascèse, une retraite en moi-même. Jamais la ville ne tendait la main vers moi; elle me laissait simplement en paix. Nous en étions convenues.

Ainsi que du fait qu'elle offrait sa protection aux productions de mon esprit ${ }^{30}$.

Ces témoignages portent à penser que le monolinguisme des premiers livres d'Ilma Rakusa s'explique précisément par ce besoin d'une limitation, d'un ancrage fort dans une langue, afin de permettre le sondage de l'espace intérieur. Car, le plurilinguisme, qui aujourd'hui constitue l'une des particularités du style d'Ilma Rakusa, a mis du temps à s'introduire dans son écriture.

\section{Le plurilinguisme dans l'écriture}

Par moments, les multiples langues de l'univers poétique et réel d'Ilma Rakusa semblent littéralement la hanter, voire l'assaillir ${ }^{31}$. Ainsi, pour décrire son identité, elle emploie l'image du tumulte d'un orchestre de voix polyphones et polyglottes jouant dans sa tête ${ }^{32}$. Or, si la trame plurilingue accompagne toute sa vie depuis sa naissance, l'éclosion de la polyglossie dans son écriture a eu lieu beaucoup plus tard. À cet égard, il faut noter qu'en règle générale il n'existe aucun lien causal entre la compétence plurilingue d'un écrivain et la réalisation de ce plurilinguisme sous forme de polyglossie littéraire ${ }^{33}$.

Dans la première période de l'œuvre de Rakusa, la présence des autres langues et cultures, par-delà les citations de textes étrangers traduits qu'on y trouve, se limite à l'utilisation de noms propres étrangers et à l'imitation de certains traits linguistiques spécifiques à d'autres langues (comme le diminutif russe). Somme toute, l'« épreuve de l'étranger $\aleph^{34}$ pratiquée par Rakusa à cette époque, si elle cherche à briser le carcan de la pureté linguistique, à inscrire des distanciations, distorsions et étrangetés dans l'idiome allemand ${ }^{35}$, n'excède pas vraiment les limites du paradigme monolingue. C'est à partir des années 1990 seulement qu'on trouve les premiers signes d'une introduction 
massive des langues étrangères dans ses textes ${ }^{36}$. Cette part plurilingue de l'écriture va s'accroître pour culminer dans son autobiographie Mehr Meer, parue en 2009.

Le plurilinguisme d'Ilma Rakusa est un plurilinguisme interne à ses textes, par opposition aux œuvres plurilingues produites par des auteurs tels que Klaus Mann ou Yoko Tawada ${ }^{37}$, où la langue d'écriture change d'une période à l'autre ou d'un livre à l'autre ${ }^{38}$. Sa langue d'écriture exclusive est bel et bien l'allemand; elle n'a écrit aucun livre dans les autres langues qu'elle maîtrise parfaitement, comme le hongrois ou le slovène, langues familiales et familières ${ }^{39}$. Selon ses propres dires, malgré l'horizon plurilingue qui est le sien, l'allemand est la seule langue dans laquelle elle arrive à exprimer les nuances stylistiques les plus subtiles ${ }^{40}$. Concernant les formes et les fonctions de son écriture plurilingue, on peut distinguer deux cas de figure, correspondant au genre lyrique, d'une part, et au genre autobiographique, d'autre part.

À l'intérieur de son autobiographie, dont le sous-titre est "passages de la mémoire " (Erinnerungspassagen) et qui raconte sa vie de manière à la fois chronologique et poétique, les langues étrangères jouent d'abord un rôle d'illustration concrète des multiples déplacements qui sont relatés dans ce livre. Dans Mehr Meer, la multiplicité des langues, lieux et cultures ne se borne pas à leur évocation abstraite ou à l'utilisation de noms propres étrangers ; elle prend corps dans la parole vivante des personnes. On y voit ainsi apparaître une bonne dizaine d'idiomes, allant du polonais de ses ancêtres, au perse entendu lors de l'un de ses derniers voyages, en passant par les langues de son enfance (hongrois, slovène, italien), l'anglais, le russe, le français, sans oublier le grec et le latin. L'usage de ces langues se limite souvent à des phrases intercalées au milieu du récit allemand; il peut se restreindre à une expression, à quelques mots, qui peuvent revenir à d'autres endroits en ponctuant le récit ; parfois on trouve quelques vers d'un poème, d'une chanson ou d'une comptine. Plutôt que de texte plurilingue, on pourrait donc parler d'éléments hétérolingues ${ }^{41}$ qui traversent un texte essentiellement germanophone. Par égard au lecteur monolingue, une traduction ou une remarque métalinguistique explicative suit généralement ces occurrences. Le point commun de presque toutes ces langues est leur référence à l'espace est - et centre-européen, l'espace dominant dans les textes d'Ilma Rakusa, cette "Kakanienne $»^{42}$ qui dit d'elle même : «L'aiguille de $[\mathrm{m}] \mathrm{a}$ boussole intérieure pointe vers l'est $»^{43}$, la direction de ses origines, somme toute.

Au-delà de l'exotisme, le plurilinguisme de Mehr Meer contribue à l'effet de réel crée par l'écriture, en transportant le lecteur à travers les langues étrangères vers les différents univers qui composent la vie d'Ilma Rakusa. L'auteur rompt ainsi avec la fiction d'un espace de communication homogène et unifié, fiction ethnocentrique propre à toute une tradition de la littérature de voyage ${ }^{44}$. La diversité linguistique, l'alternance des codes linguistiques est ici la manifestation la plus concrète de l'altérité. À l'opposé du rêve, nourri depuis toujours par la pensée occidentale, d'une langue universelle et unique $^{45}$, elle illustre la richesse que constitue la diversité des univers et des représentations inscrites dans la pluralité linguistique : "Chaque langue comporte un monde propre à elle. Et en parlant une langue, je participe de ses coutumes et particularités. A chaque fois, je suis une autre, transformée par cet intermédiaire même ", dit-elle dans l'une de ses leçons données à Dresde ${ }^{46}$. Et dans Mehr Meer, elle écrit : «Les langues étaient pour moi bien plus qu'un moyen de communication; elles étaient des mondes ayant leur lois propres comportant une sonorité spécifique ${ }^{47}$. » Avec 
les moyens de la littérature, Rakusa entend créer un univers des langues-cultures qui comporte le plus grand nombre de mondes possibles.

En outre, la pluralité des langues apparaît comme directement liée à la question de l'identité, question cruciale inscrite dans toute autobiographie. Ainsi, en parlant de son enfance, elle définit: "La diversité des langues comme diversité des identités. Oui. Dans chaque langue, je me sentais différente, et changer d'une langue à l'autre avait quelque chose de voluptueusement ludique pour moi $»^{48}$. À ce sujet, Aurélia Klimkiewicz souligne "l'impact que la pratique du bi/multilinguisme a sur la conscience de soi alors que celle-ci est continuellement traversée par la traduction, l' autotraduction ou l'auto-dialogue multilingue. ${ }^{49} \mathrm{Et}$ comme l'écrivent Schmeling et Schmitz-Emans : "Les textes linguistiquement hybrides représentent pour beaucoup d'auteurs de la littérature actuelle une forme de quête identitaire, d'une identité qui est elle-même multiple, sur le plan ethnico-culturel, social et psychique ${ }^{50}$. " Dans ce sens, on pourrait aller jusqu'à affirmer que l'« écriture de soi » pratiquée par Ilma Rakusa ne saurait être que plurilingue.

\section{La poésie comme lieu privilégie de la rencontre des langues}

21 Si dans l'autobiographie d'Ilma Rakusa, la présence des nombreuses langues autres que l'allemand prend la forme d'interjections, de citations et du discours direct, la poésie lyrique, genre que l'écrivaine affectionne le plus, donne lieu à une forme différente du plurilinguisme. Dans ses poèmes, la langue étrangère peut prendre davantage de place, acquérir une certaine autonomie jusqu'à devenir une voix indépendante, en forme de contrepoint. Voici l'un des premiers poèmes où apparaît cette forme d'écriture plurilingue, poème daté de 1991 et intitulé « Who is who?» :

Wer kann sich schon in den eigenen Kopf hineinschauen?

Vielleicht ist ein Filmorchester darin. Seufzer und

Sprache durchfluten das Gehirn. Ein Etagenwerk von

sich stapelnden Gedanken und Erinnerungen

Die Stimmen (zwei sind es immer) schälen sich aus dem

Schädel, leuchtende Signale aus der Camera obscura.

Who responds?

Who is wrong and who is right?

Stirbst du auch gerade?

Jetzt müssen wir übergehen zum indirekten Sprechen.

[...]

22 À cette pluralité des langues fait écho tout un éventail d'allusions et de citations de nature intertextuelle ${ }^{51}$, elles-mêmes souvent en langue étrangère. Si ce plurilinguisme poétique se limite à un bilinguisme allemand-anglais, complété parfois par d'autres langues, le rapport entre ces deux voix et langues n'en est que plus étroit.

La scène de la plus forte et intense confrontation entre l'allemand et l'anglais se situe dans le recueil Love after love, une suite de huit «chants d'adieu », publiée en $2001^{52}$. Voici un extrait du poème final And Venice, poème ou l'italien apparaît comme troisième langue sous forme de noms propres vénitiens :

[...]

Der Sonnenuntergang

spielt mit den Paaren 


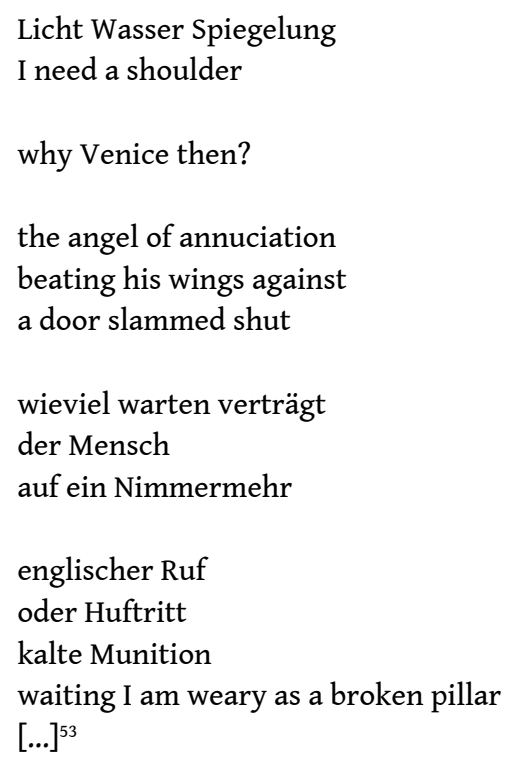

De premier abord, l'anglais, langue internationale omniprésente dans l'espace public mondial (ne serait-ce que sous forme d'un idiome rudimentaire appelé Globish ou Airport English), semble avoir un tout autre statut que les autres langues utilisées par Ilma Rakusa, langues à fort ancrage culturel, langues inscrites dans des territoires particuliers, langues parfois minoritaires. Or, l'anglais, outre l'attrait esthétique qu'il peut avoir, possède également une dimension très personnelle : d'une part, c'est l'une des langues du Trieste de son enfance, ville occupée possédant une zone angloaméricaine (c'est pour cette raison que cette langue occupe une place importante dans les livres d'Ilma Rakusa, notamment dans Mehr Meer) ${ }^{54}$. D'autre part, c'est l'une des langues de l'amour, étant la langue de l'ancien partenaire au centre du recueil Love after love.

Dans ce recueil, la confrontation entre l'anglais et l'allemand symbolise le travail de deuil suite à une rupture amoureuse. Cette confrontation prend une tonalité située entre l'élégie et la diatribe. La colère est véhiculée par un rythme rapide et une syntaxe en cascade ; la résignation par contre se traduit par un ralentissement rythmique. Entre le désir de préserver l'objet de l'amour et la nécessité de s'en défaire, entre la fusion des idiomes, correspondant à la fusion amoureuse, et l'opposition entre les langues créatrice de distance, ce va-et-vient linguistique à forte valeur cathartique, prenant parfois la forme d'un exorcisme, est l'aspect le plus intime que Rakusa a donné au plurilinguisme dans son écriture. Or, l'enchevêtrement des voix linguistiques dépasse le cadre intime et personnel pour devenir une confrontation littéraire entre le même, le principe monologique, et l'autre, symbolisé par l'autre langue, la polyphonie.

Ce corps à corps entre les langues, entre l'union et la séparation, engage des moyens formels spécifiques, comme les jeux de mots plurilingues moyennant assonances, palindromes, anagrammes, etc. A l'image de l'écriture de Rakusa en général, la matérialité du langage, la dimension rythmique et sonore des mots, s'émancipe par rapport à la dimension sémantique : «Ce n'est pas le thème qui mène au poème, mais la langue elle-même, qui se condense rythmiquement, pour passer aussitôt par le contrôle de l'ouie. $\aleph^{55}$ Approche qu'illustrent ces quelques exemples extraits de Nevermore, poème inaugural du cycle :

[...]

Remember, I care. 
I caress you.

Das Karo ist leer.

Keiner.

Nur das Geschwader tobt.

$[\ldots]$

[...]

Leave me, lover. Belagerung beendet.

Die Festung freit sich selbst.

Freit sich, lover. In frivoler Verzweiflung.

Don't cry.

Don't be shy.

Und das Pendel schwingt: Nein.

Kein Spielball mehr. Deiner Ungeduld. [... ${ }^{56}$

À certains moments, ce travail sur la matière des langues tend vers un pur plaisir des sonorités, rappelant ce témoignage de l'auteur: «Encore aujourd'hui, je m'approche des langues qui me sont étrangères par le biais de l'ouïe. C'est précisément parce que je ne comprends rien que leur sonorité prend du relief pour moi ${ }^{57}$. » De la musique avant toute chose, dixit Verlaine. Ou bien, selon Ilma Rakusa: "La musicalité plus que le $\operatorname{sen}^{58}$.»

\section{L'horizon utopique du plurilinguisme}

D'un plurilinguisme de naissance, l'ayant dotée d'au moins quatre langues différentes, Ilma Rakusa sera passée à un plurilinguisme d'écriture, présent surtout dans son œuvre de la maturité, en passant par une période de monolinguisme littéraire, correspondant à sa première période d'écriture, l'ancrage dans la langue allemande (et dans un lieu fixe) semblant avoir été nécessaire pour la formation de l'espace intérieur de la création.

Dans sa forme la plus extrême, l'usage des langues étrangères peut par moment s'approcher d'une sorte de cacophonie babélienne, explicitement recherchée par l'écrivaine. La pluralité des langues la réconcilie avec ses origines multiples et reflète la multiplicité du moi, selon son affirmation rimbaldienne que «Je est multiple». Les différentes composantes linguistiques entrent dans un rapport allant de l'opposition jusqu'à l'union, en passant par différents états tels que le rapprochement, le dialogue, la tension, le différend, etc.

Or, contrairement aux écrivains pratiquant un bi- ou plurilinguisme supratextuel, quand l'œuvre comporte plusieurs versants en plusieurs langues, l'entre-deux revendiqué par Ilma Rakusa n'est pas un véritable entre-deux linguistique. Son affirmation d'être avant tout une écrivaine germanophone n'est pas démentie par ses textes, même ceux qui sont le plus marqués par la diversité linguistique. Son work in progress, sa construction d'une identité littéraire et linguistique propre, tendant vers un plurilinguisme de plus en plus affirmé, repose certes sur une ouverture parfois radicale à l'altérité des autres langues et cultures, mais sur fond d'écriture majoritairement monolingue.

En parlant de sa poésie hétérolingue, Ilma Rakusa admet que sa réalisation concrète du plurilinguisme ne s'approche que très partiellement de son idée d'un texte babélien. Aucun de ses textes n'ose véritablement condenser toutes les langues dont elle dispose, 
la dizaine de langues contenues dans Mehr Meer étant disséminées sous forme de bribes sur un texte de plusieurs centaines de pages. Ilma Rakusa ne s'inscrit pas dans la tradition babélienne de la poésie dadaïste ou lettriste, comme l'a fait un poète comme Oskar Pastior qui pousse le mélange des idiomes jusqu'à créer un langage privé. Cependant, Ilma Rakusa se demande à juste titre quel lecteur serait réellement capable de la suivre si elle écrivait des textes mêlant l'allemand, le hongrois, le russe, le français, l'anglais, l'italien, etc. ${ }^{59}$ Le désir de multiplier les langues se heurte aux capacités limitées du public, mais aussi à celles de l'écrivain qui risque de s'épuiser dans cette conquête des langues du monde ${ }^{60}$.

C'est à ce niveau que l'écriture plurilingue est relayée par son utopie babélienne, une utopie qu'elle décrit dans ses leçons de littérature et qu'elle exprime par ce vers: "Nous parlions à la façon de Babel, avec des mots bariolés " ${ }^{61}$. Ainsi, la totalité des langues et cultures à laquelle elle aspire est avant tout une totalité idéelle, ce qu'elle appelle elle-même son "horizon utopique $»^{62}$. Dans un texte sur Rakusa, Monika Straňáková décrit cette utopie comme suit :

À travers son récit, les paysages littéraires des périphéries européennes s'unissent en un espace sans frontières, espace qu'ils n'ont jamais réellement constitué et qu'ils ne pourront probablement jamais constituer, mais dans lequel il est possible, sur le mode symbolique, de vivre le rêve d'une entente, voire s'une simultanéité culturelle et linguistique, comme le fait l'écrivaine ${ }^{63}$.

Cet espace utopique peut être considéré comme relevant d'une utopie rétrospective, comme l'un des avatars du mythe habsbourgeois, dans la mesure où la plupart des langues et cultures concernées appartiennent à l'espace de l'Empire habsbourgeois ${ }^{64}$. Son projet ne correspond-il pas finalement à la recherche d'une unité originelle qui sous-tendrait l'identité polyphonique (centre)européenne ${ }^{65}$, et qui serait à la base de sa propre identité "kakanienne "? Dans ce sens, son plurilinguisme serait finalement l'une des réalisations possibles de l'idée de Marina Tsvetaïeva, citée en exergue : «on devient poète [...] pour être non pas français, russe, etc., mais pour être tout. »

\section{NOTES}

1. Marina Tsvétaïeva, Lettre à Rainer Maria Rilke, 6 juillet 1926, in : Rainer Maria Rilke, Boris Pasternak, Marina Tsvétaïeva, Correspondance à trois : été 1926, Paris, Gallimard, 1983, p. 211.

2. Ilma Rakusa, Zur Sprache gehen. Dresdner Chamisso-Poetikvorlesungen, Dresden, Thelem, 2006, p. 7. 3. Rakusa, Zur Sprache gehen, op. cit., p. 10. «Die Grenze sensibilisiert für Vielfalt und für die Spannung zwischen Innen und Außen, zwischen Vertraut und Fremd, zwischen Nah und Fern. Für diese Sensibilisierung bin ich dankbar. Denn zweifellos hat sie dazu beigetragen, daß ich zur schreibenden Grenzgängerin und Übersetzerin geworden bin. Der Grenzverkehr zwischen Sprachen und Staaten wurde zu meiner Lebensschule, die Reibung zwischen dem Eigenen und dem Fremden zum künstlerischen Stimulus. Ich erlebte die Grenze in ihrer (produktiven) Doppeldeutigkeit : nämlich als Schranke und Brücke in einem. » Toutes les traductions de textes d'Ilma Rakusa cités dans cette contribution ont été établies par moi-même. Par rapport à 
l'ambivalence de la frontière, on peut également consulter le livre de Régis Debray, Éloge des frontières, Paris, Gallimard, 2010.

4. Rakusa, Zur Sprache gehen, op. cit., p. 9.

5. Ibid., p. 13.

6. Ibid., p. 31 : «Es gibt Tage, da führe ich Selbstgespräche in fünf Sprachen und spüre, daß auch das Schreiben sich fünfsprachig gebärden möchte. Nimm von allem das Beste [...] und schaff dir dein eigenes, multilingual changierendes Idiom. "

7. Sur le rapport entre Babel et la littérature, voir notamment Violaine Houdart-Merot (dir.), Écritures babéliennes, Berne, Lang, 2006.

8. Zur Sprache gehen, op. cit., p. 9.

9. Ibid., p. 7.

10. «Oui, je n'ai qu'une langue, or ce n'est pas la mienne », Jacques Derrida, Le Monolinguisme de l'autre, ou la prothèse d'origine, Paris, Galilée, 1996, p. 15.

11. Rappelons que c'est la ville de Claudio Magris, l'auteur de l'étude classique intitulée Il mito asburgico nella letteratura austriaca moderna (1963), publiée en français sous le titre Le Mythe et l'empire dans la littérature autrichienne moderne, Paris, Gallimard, 1963.

12. Rakusa, «'Daheim im Dazwischen'. Dankesrede », in : Börsenblatt für den Deutschen Buchhandel, Frankfort/Main, 7.4.1998. Il s'agit du discours de remerciement lors de la remise du Prix de l'Entente Européenne de la Foire du Livre de Leipzig.

13. Rakusa, Mehr Meer. Erinnerungspassagen, Graz-Vienne, Droschl, 2009 ; version française : La Mer encore. Passages de la mémoire, trad. Patricia Zurcher, Lausanne, Éditions d'en bas, 2012.

14. Ibid., p. 86.

15. Rakusa, Zur Sprache gehen, op. cit., p. 31: «Auf welchem Sprachboden bewege ich mich, was unterscheidet diese von der anderen Sprache, usw. Ich lernte von klein auf vergleichen, sondieren, abtasten. Ich verlernte den Glauben an ein Einziges, Absolutes, Tragfähiges. »

16. D'après ses propres dires, cf. Mehr Meer, op. cit., p. 107.

17. Cela la rapproche d'Elias Canetti qui n'a appris l'allemand qu'à huit ans, comme quatrième langue également.

18. Sans parler du fait que la Suisse est un pays qui compte pas moins de quatre langues officielles; toutefois, ce cadre plurilingue du pays de résidence ne semble pas réellement s'intégrer à l'univers littéraire d'Ilma Rakusa.

19. Rakusa, « Der Tumult des Kopforchesters », in : Uwe Pörksen, Bernd Busch (dir.), Eingezogen in die Sprache, angekommen in der Literatur. Positionen des Schreibens in unserem Einwanderungsland, Göttingen : Valerio 8, Wallstein Verlag, 2008, (p. 76-80), p. 76 : « Deutsch ist mir nicht zugefallen, es verdankt sich einem Akt der Anstrengung, der mir indes viele Türen öffnete. »

20. Rakusa, Zur Sprache gehen, op. cit., p. 8: "Mein Ohr war auf Sprachen sensibilisiert: sie markierten Reichtum und Differenz. »

21. Rakusa, " Meine Sprache », in: Klaus Amann, Fabjan Hafner (dir.), Mein Paradies und andere Orte der Begegnung, Graz, Styria, 2003, (p. 97-104), p. 102.

22. Rakusa, Wie Winter. Gedichte, Zurich, Edition Howeg, 1977.

23. Rakusa, Mehr Meer, op. cit., p. 76.

24. Elle-même se décrit ainsi dans Zur Sprache gehen, op. cit., p. 16.

25. Ibid., p. 9. Sur le rapport entre plurilinguisme et refus de l'enracinement voir aussi Axel Gasquet, Modesta Suárez (dir.), Écrivains multilingues et écritures métisses. L'hospitalité des langues, Clermont-Ferrand, Presses Universitaires Blaise Pascal, 2007, p. 9.

26. Rakusa, Mehr Meer, op. cit., p. 36 : «Mit zunehmendem Alter frage ich mich, wer ich bin. / Koffer, auch wenn sie voller farbig-fröhlicher Aufkleber sind, geben darauf keine Antwort. / Koffer tun weh.»

27. Ibid., p. 87 : «Weiter, immer weiter, diese Melodie kannte ich schon. Nur über das Warum war ich mir im unklaren. Mich hatte auch keiner je gefragt. » Le début peut effectivement faire 
penser au lied « Das Wirtshaus» du Voyage d'hiver (« Nun weiter denn, nur weiter, / Mein treuer Wanderstab! »).

28. Ibid., p. 107 : « Nach drei Sprachen, die ich zuvor erlernt hatte, war diese vierte Fluchtpunkt und Refugium. Hier wollte ich mich niederlassen, hier baute ich mir mein Haus. »

29. Ibid., p. 184 : «Ging es denn um Stabilität? Um ein Gegengift gegen die Nomadenhaftigkeit meiner Kindheit, mit ihren Ortswechseln, Umzügen, Unsicherheiten? Setzte ich mir selber Grenzen? Um andere, innere Räume zu erkunden?»

30. Ibid., p. 221 : «Damals kam mir der Gedanke, Zürich verlange von mir Askese, einen Rückzug in mich selbst. Nie streckte es die Hand nach mir aus; es ließ mich einfach in Ruhe. Darauf einigten wir uns. / Und daß es meinen Kopfgeburten Schutz bot. »

31. Rakusa, « Meine Sprache », op. cit., p. 98 et p. 102.

32. Rakusa, « Der Tumult des Kopforchesters », Zur Sprache gehen, op. cit., p. 77.

33. Cf. Georg Kremnitz, Mehrsprachigkeit in der Literatur. Wie Autoren ihre Sprachen wählen. Aus der Sicht der Soziologie der Kommunikation, Vienne, Edition Praesens, 2004, p. 254.

34. D'après l'étude classique d'Antoin Berman, L'épreuve de l'étranger. Culture et traduction dans l'Allemagne romantique, Paris, Gallimard, 1984.

35. Cf. Rakusa, "Vorstellungsrede in der Deutschen Akademie für Sprache und Dichtung », in : Jahrbuch der Deutschen Akademie für Sprache und Dichtung, 1996. Göttingen, Wallstein, 1997, (p. 168-170), p. 170.

36. Le recueil Ein Strich durch alles. Neunzig Neunzeiler (Francfort/Main, Suhrkamp, 1997) comporte un poème intégralement écrit en anglais et des éléments plurilingues dans d'autres poèmes. Dans le cas du recueil Les mots / morts (Zurich, Edition Howegg, 1992) et des poèmes " Some blackness » et «We shall dance » (in : SMS-Lyrik, 160 Zeichen Poesie, éd. Anton G. Leitner. Munich, dtv, 2002, p. 52 et 59), seul le titre est en langue étrangère.

37. Pour ne citer que ces deux exemples d'écrivains germanophones plurilingues. Sur Mann, voir Susanne Utsch, « Der exilbedingte Sprachwechsel von Klaus Mann - im Fokus von Sprach- oder Literaturwissenschaft? Ein Plädoyer für interdisziplinäre Grenzüberschreitungen », in : Dieter Heimböckel et alii. (dir.), Zwischen Provokation und Usurpation. Interkulturalität als (un)vollendetes Projekt der Literatur- und Sprachwissenschaften, Munich, Fink, 2010, p. 275-305 ; sur Tawada voir Bernard Banoun et Linda Koiran (dir.), L'Oreiller occidental-oriental de Yoko Tawada, Études germaniques, 65-63, juillet-septembre 2010.

38. Par rapport à cette distinction, voir Kremnitz, op. cit.

39. Sur le plan sociolinguistique, le prestige de l'allemand comme langue littéraire entre également en jeu, même s'il n'est pas avéré que ce prestige ait joué un rôle dans le choix effectué par Rakusa.

40. Rakusa, « Vorstellungsrede », op. cit., p. 169-170.

41. Au sujet du qualificatif de hétérolingue, voir Myriam Suchet, Outils pour une traduction postcoloniale, Paris, éditions des archives contemporaines, 2009.

42. « Kakanierin », dérivé de «K.(a)u.(nd)K.(a) », surnom donné à la monarchie austro-hongroise.

43. Ibid., p. 23 : «Die innere Kompaßnadel zeigt nach Osten. »

44. Cf. Edzard Obendiek, Der lange Schatten des babylonischen Turmes. Das Fremde und der Fremde in der Literatur, Göttingen, Vandenhoeck\&Ruprecht, 2000, p. 9.

45. Cf. Umberto Eco, La Recherche de la langue parfaite dans la culture européenne, Paris, Le Seuil, 1994.

46. Rakusa, Zur Sprache gehen, op. cit., p. 19 : «Jede Sprache bedeutet eine eigene Welt. Und indem ich sie spreche, nehme ich teil an ihren Sitten und Charakteristiken. Ich bin jedesmal eine andere, verwandelt durch das Medium selbst. »

47. Rakusa, Mehr Meer, op. cit., p. 228: "Sprachen waren für mich mehr als nur Verständigungsmittel ; sie waren eigengesetzliche Welten mit einem spezifischen Klang. » 
48. Rakusa, «Meine Sprache », op. cit., p. 99 : «Sprachenvielfalt als eine Vielfalt von Identitäten. Ja. Ich fühlte mich in jeder Sprache anders, und es hatte etwas lustvolle Spielerisches für mich, von einer Sprache zur anderen zu wechseln. »

49. Aurélia Klimkiewicz, «Faire l'épreuve de soi au travers de l'auto-dialogue multilingue : à propos du commentaire métalangagier dans la littérature migrante », in : Francis Mus et Karen Vandemeulebroucke (dir.), La Traduction dans les cultures plurilingues, Arras, Artois Presses Université, 2011 (p. 23-31), p. 26-27.

50. Manfred Schmeling, Monika Schmitz-Emans (dir.), Multilinguale Literatur im 20. Jahrhundert, Würzburg, Königshausen \& Neumann, 2000, p. 19: « Hybridsprachliche Texte sind [...] für viele Autoren der Gegenwartsliteratur Formen der Suche nach einer Identität, die selbst multipel ist : auf ethnisch-kultureller, sozialer und psychischer Ebene. » Traduction DW.

51. En l'occurrence, on peut y trouver une citation de la pièce Abendlandleben oder Apollinaires Gedächtnis de Gisela von Wysocki, créée en 1999 et éditée par Suhrkamp. Les deux premiers vers, légèrement modifiés, sont extraits de la pièce.

52. Rakusa, Love after Love. Acht Abgesänge, Francfort/Main, Suhrkamp, 2001. Comme indiqué plus haut, le recueil Ein Strich durch alles, comporte, p. 67, un poème en langue anglaise.

53. Ibid., p. 52.

54. Voir Rakusa, « Meine Sprache », op. cit., p. 99.

55. Ibid., p. 98 : «Nicht ein Thema führt zum Gedicht, sondern die Sprache selbst, die sich rhythmisch ballt, um alsbald die Kontrolle des Ohrs zu passieren. »

56. Rakusa, « Nevermore », Love after love, op. cit., p. 12 et p. 13.

57. Rakusa, Zur Sprache gehen, op. cit., p. 114. « Noch heute nähere ich mich fremden Sprachen mit dem Ohr an. Gerade weil ich nichts verstehe, wird mir ihre Klanglichkeit plastisch. »

58. Ibid. : « Mehr Klang als Sinn. »

59. Ibid., op. cit., p. 16.

60. Cf. ses propos pendant la discussion faisant suite à « Meine Sprache », op. cit., p. 112-113.

61. Rakusa, Ein Strich durch alles, op. cit., p. 87 : « wir sprachen babylonisch kunterbunt ».

62. Ibid.

63. Straňáková, Monika, «Literatur als fremde Sprache - fremde Sprache(n) in der Literatur. Anmerkungen zum mehrsprachigen Schreiben von Irena BreŢná und Ilma Rakusá », in : Michaela Bürger-Koftis, Hannes Schweiger, Sandra Vlasta (dir.), Polyphonie - Mehrsprachigkeit und literarische Kreativität, Vienne, Edition Praesens, 2010, (p.390-405), p.391: «Die Literaturlandschaften europäischer Peripherien fügen sich in ihrer Darstellung zu einem grenzenlosen Raum zusammen, der sie nie wirklich waren und zu dem sie womöglich auch nie werden können, in dem man aber, wie die Dichterin, den Traum eines kulturellen und sprachlichen Miteinanders, ja Simultaneität, symbolische leben kann. » Traduction DW.

64. Cf. Walter Schmitz, «Das Ich im Netz der Sprache. Zu Ilma Rakusas Schreiben », in : Rakusa, Zur Sprachen gehen, op. cit., (p. 193-251), p. 198.

65. Cf. Johann Strutz, Peter V. Zima (dir.), Literarische Polyphonie. Übersetzung und Mehrsprachigkeit in der Literatur. Tübingen, Narr, 1996, p. 9-10. 


\section{RÉSUMÉS}

Née en 1946 en Slovaquie, d'un père slovène et d'une mère hongroise, Ilma Rakusa se décrit ellemême comme "rien d'autre qu'une écrivaine de langue allemande vivant à Zurich ». Or, rares sont les auteurs germanophones à posséder un arrière-fond linguistique et culturel aussi riche. N'ayant appris l'allemand que comme quatrième langue, la diversité linguistique est l'horizon quasi naturel de sa vie et de son travail comme écrivaine, traductrice et essayiste. Dans son écriture, le plurilinguisme se manifeste sous plusieurs formes: autobiographique, poétique et utopique. À partir notamment de son autobiographie Mehr Meer et de son recueil de poèmes Love after love, cet article se propose d'examiner la dimension plurilingue de son œuvre.

Born in Slovakia in 1946 to a Slovenian father and a Hungarian mother, Ilma Rakusa describes herself as "nothing but a German language writer living in Zurich." However, few other German language authors have such a rich linguistic and cultural background. Having learned German only as a fourth language, the linguistic diversity is the almost natural horizon of her life and her work as a writer, translator and essayist. In her works multilingualism appears in several forms: an autobiographical, a poetic and a utopian forms. Based on a reading of her autobiography Mehr Meer, her poems Love after love and other texts this paper aims at analysing the multilingual dimension of Ilma Rakusa's works.

Ilma Rakusa wurde 1946 in der Slowakei als Kind eines Slowenen und einer Ungarin geboren und beschreibt sich selbst als „nichts anderes [...] als eine deutschsprachige Schriftstellerin, die in Zürich lebt." Kaum ein anderer deutschsprachiger Autor besitzt jedoch einen so reichen sprachlichen und kulturellen Hintergrund wie sie. Da sie das Deutsche erst als vierte Sprache erlernt hat, stellt die Sprachenvielfalt gleichsam den natürlichen Horizont ihres Lebens und ihrer Arbeit als Schriftstellerin, Übersetzerin und Essayistin dar. Mehrsprachigkeit ist in ihrem Werk in mehreren Formen präsent : einer autobiographischen, einer poetischen und einer utopischen. Auf der Grundlage ihrer Autobiographie Mehr Meer, ihres Gedichtbandes Love after love und anderer Texte soll die mehrsprachige Dimension von Rakusas Werk untersucht werden.

\section{INDEX}

Mots-clés : plurilinguisme

\section{AUTEUR \\ DIRK WEISSMANN}

Université Paris-Est Créteil 